\title{
Increasing significance of external quality assurance in higher education: current strategies applied by European agencies
}

\section{Sánchez Chaparro, Teresa and Gómez Frías, Víctor}

Department of Engineering Management, Universidad Politécnica de Madrid, Spain

\begin{abstract}
Quality assurance of higher education programmes and institutions has been one of the cornerstones of the Bologna process since its creation. However, after more than one decade of implementation of the European quality assurance framework, many national systems are suffering from the so-called "evaluation fatigue”. From a thorough revision of key sectorial sources, this paper identifies a number of strategies currently being tested by European quality assurance agencies aimed at increasing significance and reducing bureaucracy of external quality assurance processes.
\end{abstract}

Keywords: External quality assurance; Quality assurance agencies; Impact; Higher Education 


\section{Introduction}

Quality assurance of higher education programmes and institutions has been one of the cornerstones of the Bologna process since its creation. Quality assurance has the primary mission of building mutual trust among Higher Education Institutions and other stakeholders all over Europe (Bergan, 2011). Together with other structural elements, such as the European Qualification Framework and the European Credit Transfer System, quality assurance constitutes the foundation for a smooth mobility of students and graduates along the EHEA (EHEA, 2005).

The particular European approach to quality assurance is specified in a key document, the "Standards and Guidelines for Quality Assurance in the European Higher Education Area" or ESG (ENQA et al., 2015). A first version of the ESG was initially presented and approved at the Bergen Ministerial Conference in 2005 (EHEA, 2005). An updated version of the standard was released and approved ten years later at the Ministerial Conference in Yerevan (EHEA, 2015). This document reflects a consensus among all the organisations concerned with the promotion and implementation of quality assurance in the European Higher Education Area.

The ESG makes a distinction between "internal quality assurance" (IQA) -the quality assurance (QA) practices and strategies put in practice by higher education institutions to ensure their own educational quality- and "external quality assurance" (EQA) -the strategies and processes implemented by external bodies (a.k.a quality assurance agencies).

Many of these bodies have been developed since the beginning of the century and are now well established in the majority of EHEA countries. ENQA -the European Network for Quality Assurance in Higher Education- is a membership association whose members are the external quality assurance bodies officially recognized in each country. As of February 2018, ENQA included 52 members in 29 EHEA countries.

While the ESG makes it explicit that the major responsibility of QA lies within HEIs (IQA), EQA and QA agencies have a major role as control and enhancement actors. They assist the institutions in implementing their internal quality assurance systems and, in certain occasions, play a leading role in the introduction of quality assurance innovations.

The development and acceptation of these external bodies have not been simple. Stakeholders, and academics in particular, tend to resist any quality procedures that are perceived as disconnected of their primary teaching and research activity (Harvey and Williams, 2010; Newton, 2002). After a number of evaluation cycles, many national systems are suffering from the so-called "evaluation fatigue" (Schwarz and Westerheijde, 2007). The recent economic crisis has increased the sensitivity of the sector, the government and the general public towards a reduction of bureaucratic burden and a 
rationalization of resources, which has even lead to the suppression or merger of quality assurance agencies in various countries (Walsh, 2012).

In this context, quality assurance agencies have felt the need to explore new strategies aimed at increasing the added value of EQA processes over the core teaching and learning activity. After explaining the most recent developments introduced by the 2015 version of the ESG, this paper takes stock of the most recent strategies adopted by quality agencies in order to rationalize resources and increase significance of their EQA processes all over Europe.

\section{Major changes introduced by the updated 2015 version of the ESG}

While the nature and structure of the ESG have remained essentially the same, the updated 2015 version introduces new core notions with the intention of increasing the impact and significance of the standard. The most substantial changes introduced are:

- The student-centred learning (SCL) paradigm, a learning approach that puts the learner at the centre (Boyer, 1990; Machemer and Crawford, 2007), has been adopted as an explicit requirement.

- The scope of quality assurance has been expanded to include the full student life cycle (such as student admission, progression, recognition and certification). With regard to institutional recognition practices, the standard makes it explicit that they should be in line with the principles of the Lisbon Recognition Convention and rely on cooperation with the national ENIC-NARIC centres.

- New requirements on transparency and management of information have been introduced, such as the publication by the agencies of the "full" reports by the experts or the collection, analysis and use of relevant information by the higher education institutions for the effective management of their programmes.

\section{Current strategies of QA agencies to increase significance of their EQA processes}

After a number of evaluation cycles, many quality assurance agencies have undergone reforms aimed at increasing efficiency and significance of their national external quality assurance frameworks and at incorporating the new criteria stablished by the new version of the ESG.

The trends explained below have been identified after a thorough revision of key sectorial sources, such as the communications, posters and key note speeches at the ENQA general assemblies and the trends and developments presented at the largest annual gathering of the 
European higher education quality assurance, the European Quality Assurance Forum (EQAF).

\subsection{Diversity of approaches and objectives}

In the past, many national external quality assurance frameworks have been developed following two alternative monolithic approaches: institutional versus programme-oriented. Similarly, quality assurance agencies have frequently positioned themselves in the control versus enhancement dichotomy and tend to consider that these two objectives are difficult to combine and belong to different organizational cultures (Sánchez et al., 2011).

Agencies are now breaking these binary schemes. Recent reforms all over Europe have set up new EQA frameworks that apply a diversity of approaches and objectives. This is the case of the new EQA model recently implemented in Sweden, which comprises institutional reviews, programme evaluations and accreditations and thematic evaluations and focus both on output and internal processes (Boström and Kettis, 2016). The Frenchspeaking part of Belgium provides another example, as the system is currently shifting from a purely programmatic enhancement-oriented approach to a combination of institutional and programme assessment and the introduction of some accountability elements (Aubert and Duykhaerts, 2017). In the same vein, the new review model currently being implemented in Ireland combines multiple objectives (effectiveness, accountability and enhancement) and multiple dimensions (institution, system and thematic) (Lynch, 2017).

\subsection{Risk based approach}

Several agencies in Europe have recently reported to be in the process of developing riskbased approaches. Under this philosophy, a system of key indicators that are carefully monitored is used to predict the outcome of external quality assurance reviews. Institutions are applied EQA processes of different depth and intensity depending on the result of the risk assessment. This practice tries to address the unavoidable "evaluation fatigue" by the institutions and, at the same time, is bound to increase effectiveness of the EQA system.

In the field of quality assurance, two countries -Norway and United Kingdom- have headed this trend that is currently being explored by many European countries, even though the development of national systems of risk indicators are not yet fully tested and developed (Griffiths and Halford, 2015; Lund, 2018).

Several practical challenges to the application of risk-based approaches for regulatory purposes have been documented in literature (Black, 2005 and 2008; Black and Baldwin, 2012; Rothstein et al., 2006). Indeed, previous experiences outside Europe have been problematic. The Australian agency (TEQSA) attempted a risk-based approach to quality assurance in higher education in 2012. The initial approach lead to strong complaints from 
the sector and resulted in the publication of a simplified and more robust regulatory risk framework (TEQSA, 2012 and 2016).

\subsection{Focus on enhancement}

A clear trend in external quality assurance is the effort by many QA agencies to include an enhancement element into their external quality assurance procedures. Agencies put in place various strategies, which normally involve the introduction of a more flexible and conversational evaluation format that substitutes or complements a more formal step-bystep control-oriented evaluation or accreditation exercise. This increased flexibility enables to liberate resources and concentrate in substantial aspects (student-centred learning, sectorspecific aspects, societal implications), which is bound to increase the impact of the EQA processes.

An example is the so-called "appreciative approach" implemented in Flanders since 2014, a flexible evaluation scheme whose most relevant characteristics are contextualization, the postponement of judgements, the focus on relevant aspects, the continuous dialogue and the co-creation of insights (Aerden et al., 2016).

In Finland, the QA agency (FINEEC), with a long tradition of enhancement-led institutional evaluations and after two in-depth reviews of institutional audits, has launched in 2018 a new audit model. The new approach is built on an idea of a higher education institution as an open system and a learning organization. The focus is on student centred learning and on the societal impact of higher education, including the interaction with stakeholders at various levels (FINEEC, 2017).

Another example is the "critical friend" approach developed in the field of higher music education. Within this philosophy, annual visits by "critical friends" (external experts that are considered to be an international authority with regard to the content of the programmes reviewed) are combined with a lighter version of regular review visit by the quality assurance agency. This experimental approach has shown to be effective with regards to reducing the bureaucratic load of higher education institutions and creating a stronger involvement of teachers in quality assurance processes. Indeed, the "critical friend" has been said to act as a proxy, a trained peer-specialist that speaks the same language as those working and studying in the institutions and is bound to bridge the gap between quality assurance, teachers and students (Prchal and Messas, 2016).

\subsection{Information management and analytics}

Increasingly, agencies are developing analytic capacities that enable them to produce reports and information that are useful for the enhancement and development of national higher education systems. Specifically, agencies are implementing this analytic dimension through the development of quantitative studies and indicators at the system and 
institutional level, which contribute to build evidence-based decision and management systems. An example is the assessment of the research and teaching activities of university departments conducted by the Catalan Quality Assurance Agency (AQU-Catalunya) using quantitative research available in a public information system compiling key information of all universities in Catalonia. Within this experience, AQU-Catalunya has conceived a reporting system to facilitate the accessibility and interpretation of the data by higher education institutions (Prades et al., 2015). In a similar vein, the French accreditation body for engineering programmes (Commission des Titres d'Ingénieur, CTI) has developed, in collaboration with its stakeholders, a set of public indicators which mean to characterize a particular HEI. The main objective of this initiative, in place since 2013, is to provide meaningful and trusted information to the general public regarding the HEIs and their accredited programmes (CTI, 2017).

\section{Conclusions}

After more than one decade of implementation of the European quality assurance framework, it seems that practices are evolving in the external quality assurance sector. This paper, which is part of a wider reflexion from the authors on quality assurance significance and impact, has presented a selection of strategies currently being tested by European quality assurance agencies aimed at increasing significance and reducing bureaucracy of external quality assurance processes.

The application of diversified risk-based approaches seems to be one of the cornerstones of this new trend. The modulation of the intensity of EQA procedures in relation to the perceived risks saves resources and time, which can then be devoted to the introduction of an enhancement dimension and a focus on substantial aspects (such as student centred learning or societal aspects). However, the estimation of risks is a difficult task and some of the most advanced QA agencies are still trying to fine-tune their risk assessment capabilities.

Indeed, the application of risk-based approaches needs the development of powerful analytic capacities, requirement which is, incidentally, consistent with the increased focus on transparency and information management introduced by the 2015 version of the ESG. It also requires the careful consideration of some well documented challenges and difficulties. Further reflexion is required to really assess the feasibility of this promising approach to the quality assurance sector. 


\section{References}

Aarden, A. et al. (2016). NVAO's Appreciative Approach, a new philosophy to strengthen internal and external quality assurance. 11th European Quality Assurance Forum 17-19 November.

Aubert, A. and Duykaerts, C. (2017). How to get ready for change? 12th European Quality Assurance Forum Responsible QA - committing to impact. Latvia 23-25 November.

Black, J. (2005). The emergence of risk-based regulation and the new public management in the United Kingdom. Public Law, (Autumn). pp. 512-549.

Black, J. (2008). Risk Based Regulation: Choices, Practices and Lessons Being Learned. In: Risk and Regulatory Policy: Improving the Governance of Risk. Organization for Economic Co-operation and Development, Paris.

Bergan, S. (2011). Building Trust. Quality and Trust: At The Heart of What We Do. A Selection of Papers From The European Quality Assurance Forum. Bruxelles: European University Association. pp. 52 - 57.

Boyer, E.B. (1990). Scholarship Reconsidered. Priorities of the Professoriate. New York: The Carnegie Foundation for the Advancement of Teaching.

European Higher Education Area (2005). Communique of the Conference of Ministers responsible for Higher Education 2005. Bergen, Norway. EHEA.

European Higher Education Area (2015). Communique of the Conference of Ministers responsible for Higher Education 2015. Yerevan, Armenia. EHEA.

Boström,T. and Kettis, A. (2016). Introducing a new national QA system in Sweden. A discussion on opportunities and challenges. 11th European Quality Assurance Forum. 17-19 November.

CTI (2017). CTI's certified data, an enhancement tool for quality culture. $8^{\text {th }} E N Q A$ General Assembly, Sèvres, 26-27 October.

ENQA, ESU, EUA, EURASHE (2015). Standards and Guidelines for Quality Assurance in the European Higher Education Area (ESG). Brussels, Belgium.

FINEEC (2017). FINEEC's audit model promotes societal impact. $8^{\text {th }}$ ENQA General Assembly, Sèvres, 26-27 October.

Griffiths, A. and Halford, E. (2015). Zen and the art of risk assessment: what are the implications of a system of risk- based quality assurance for higher education in England? 10th European Quality Assurance Forum, London 19-21 November.

Harvey, L. and Williams, J. (2010). Fifteen Years of Quality in Higher Education: (Part Two). Quality in Higher Education. 16(2). 81-113. 
Lund, O. (2018). Using risk analysis and indicators to assure programme quality in institutional reviews. Institutional and Programme Reviews: Towards New Combinations, ECA-NVAO Seminar, The Hague, 1 February.

Lynch, O. (2017). A new model for external review of quality in a diverse higher education area. 12th European Quality Assurance Forum Responsible QA - committing to impact. Latvia 23-25 November.

Machemer, P.L. and Crawford, P. (2007). Student Perceptions of Active Learning in a Large Cross-Disciplinary Classroom'. Active Learning in Higher Education, 8:1, pp.930.

Newton, J. (2002). Views from below: academics coping with quality. Quality in higher education. 8(1). 39-61.

Nuffic (2017). FAIR report. The Hague, Netherlands.

Prades, A. (2015). Big (and small) data meets quality assurance. 10th European Quality Assurance Forum, London 19-21 November.

Prchal, M. and Messas, L. (2016). How to make quality assurance processes more meaningful to teaching staff - a proposal from the field of music. 11th European Quality Assurance Forum 17-19 November.

Rothstein, H.F., Irving, P., Walden, T. and Yearsley, R. (2006). The Risks of Risk-Based Regulation: Insights from the environmental policy domain. Environment International, vol 32, no. 8, pp. 1056-1065.

Sánchez Chaparro,T., Duykhaerts, C., Carette, G., Coessens, M. and Remaud, B. (2011). Combining evaluation and accreditation cultures: A collaboration between AEQES and CTI for the joint evaluation and accreditation of civil engineering programmes in the French community of Belgium in 2012-2013. INQAAHE seminar on Connecting European and Global Experiences, Brussels, 4-5 December.

Schwarz, S. and Westerheijden, D. F. (Eds.) (2007). Accreditation and Evaluation in the European Higher Education Area. Springer.

TEQSA (2012). Regulatory Risk Framework. February .

TEQSA (2016). Risk Assessment Framework. February.

Walsh, P. (2012). The Qualifications and Quality Assurance Authority of Ireland (QQAI) an amalgamated agency responsible for overseeing changes in the qualifications and quality assurance of Irish further and higher education. Presentation to CHEA International Seminar, Washington DC, 26 January. 